\title{
Temporal and spatial patterns of serologic responses to Plasmodium falciparum antigens in a region of declining malaria transmission in southern Zambia
}

Tamaki Kobayashi $i^{*}$, Sandra Chishimba², Timothy Shields ${ }^{3}$, Harry Hamapumbu ${ }^{2}$, Sungano Mharakurwa ${ }^{2,3}$, Philip E Thuma², Gregory Glass ${ }^{3}$ and William J Moss ${ }^{1,3}$

\begin{abstract}
Background: Critical to sustaining progress in malaria control is comprehensive surveillance to identify outbreaks and prevent resurgence. Serologic responses to Plasmodium falciparum antigens can serve as a marker of recent transmission and serosurveillance may be feasible on a large scale.

Methods: Satellite images were used to construct a sampling frame for the random selection of households enrolled in prospective longitudinal and cross-sectional surveys in two study areas in Southern Province, Zambia, one in 2007 and the other in 2008 and 2009. Blood was collected and stored as dried spots from participating household members. A malaria rapid diagnostic test (RDT) was used to diagnose malaria. An enzyme immunoassay (EIA) was used to detect IgG antibodies to asexual stage P. falciparum whole parasite lysate using serum eluted from dried blood spots. The expected mean annual increase in optical density (OD) value for individuals with a documented prior history of recent malaria was determined using mixed models. SatScan was used to determine the spatial clustering of households with individuals with serological evidence of recent malaria, and these households were plotted on a malaria risk map.

Results: RDT positivity differed markedly between the study areas and years: $28 \%$ of participants for whom serologic data were available were RDT positive in the 2007 study area, compared to $8.1 \%$ and $1.4 \%$ in the 2008 and 2009 study area, respectively. Baseline antibody levels were measured in 234 participants between April and July 2007, 435 participants between February and December 2008, and 855 participants between January and December 2009. As expected, the proportion of seropositive individuals increased with age in each year. In a subset of participants followed longitudinally, RDT positivity at the prior visit was positively correlated with an increase in EIA OD values after adjusting for age in $2007(0.261, p=0.003)$ and in $2008(0.116, p=0.03)$. RDT positivity at the concurrent visit also was associated with an increase in EIA OD value in 2007 (mean increase $0.177, p=0.002$ ) but not in $2008(-0.063, p=0.50)$. Households comprised of individuals with serologic evidence of recent malaria overlapped areas of high malaria risk for serologic data from 2009, when parasite prevalence was lowest.

Conclusions: Serological surveys to whole asexual P. falciparum antigens using blood collected as dried blood spots can be used to detect temporal and spatial patterns of malaria transmission in a region of declining malaria burden, and have the potential to identify focal areas of recent transmission.
\end{abstract}

\footnotetext{
* Correspondence: tkobayas@jhsph.edu

'Department of Epidemiology, Johns Hopkins Bloomberg School of Public

Health, Baltimore, MD, USA

Full list of author information is available at the end of the article
}

\section{Biomed Central}

(c) 2012 Kobayashi et al.; licensee BioMed Central Ltd. This is an Open Access article distributed under the terms of the Creative Commons Attribution License (http://creativecommons.org/licenses/by/2.0), which permits unrestricted use, distribution, and reproduction in any medium, provided the original work is properly cited. 


\section{Background}

Increased funding for malaria control and elimination has led to implementation of comprehensive control programmes and concomitant reductions in the burden of malaria in many parts of sub-Saharan Africa [1,2]. Zambia has been a model country for malaria control within sub-Saharan Africa and has achieved a significant decline in the burden of malaria [3,4]. Zambia's national malaria control programme includes provision of artemisinin-based combination therapy, distribution of insecticide-treated nets, indoor residual spraying in urban and peri-urban areas, and intermittent preventive treatment of pregnant women [3,5]. By 2008, the prevalence of parasitaemia and severe anaemia in children between six and 59 months of age decreased by $53 \%$ and $68 \%$, respectively, compared with levels in 2006 [3]. In April 2009, the World Health Organization announced that Zambia reached the 2010 Roll Back Malaria target of greater than $50 \%$ reduction in malaria mortality compared to levels in 2000 [6].

Despite this impressive achievement, the incidence of malaria increased in five of nine provinces of Zambia in $2010[4,7]$. The greatest resurgence occurred in Eastern and Luapula Provinces, where the number of reported cases of malaria doubled from levels in 2008 [4]. Such trends highlight the challenge of sustaining effective malaria control. Critical to such control is effective surveillance to identify outbreaks, target control efforts and prevent resurgence. Serologic responses to Plasmodium falciparum can serve as a proxy measure of malaria transmission [8-13] and may be a useful tool for enhanced surveillance in the pre-elimination phase of malaria control. Measurement of antibodies to single parasite antigens such as MSP- $1_{19}$ and AMA-1 identified infection within the previous four months among children younger than six years of age in The Gambia [14]. Serologic surveillance may be feasible on a large scale using blood collected on filter paper [15] or oral fluid samples [16,17]. IgG antibody levels to whole, asexual $P$. falciparum lysate were measured by enzyme immunoassay in two community-based cohorts in southern Zambia to assess the utility of serological surveys to identify temporal and spatial patterns of recent malaria transmission in a region with declining malaria burden in southern Zambia.

\section{Methods}

\section{Study site}

The study was conducted in two sites within the catchment area of Macha Hospital in Choma District, Southern Province, Zambia between April 2007 and December 2009. Macha Hospital is located approximately $70 \mathrm{~km}$ from the nearest town of Choma on a plateau at an altitude of approximately 1,100 meters above sea level and in a habitat characterized as Miombo woodland. A single rainy season, lasting from approximately December through March, is followed by a cool, dry season from April to July, and a hot, dry season from August to November. The catchment area is populated by traditional villagers living in small, scattered homesteads. Anopheles arabiensis is the primary vector responsible for malaria transmission [18], which peaks during the rainy season. The study site in 2007 consisted of a $525 \mathrm{~km}^{2}$ region to the east of Macha Hospital (Figure 1). In 2008 and 2009, the study site was shifted to a $575 \mathrm{~km}^{2}$ area west of the 2007 study site (Figure 1).

The Southern Province of Zambia historically had hyperendemic transmission of P. falciparum [19]. More recently, the entomological inoculation rate for $A n$. arabiensis was estimated to range from 1.6 to 18.3 infective bites per person per season [18]. Zambia introduced artemetherlumefantrine as first-line anti-malarial therapy in 2002, which reached the Macha Hospital catchment areas in 2004, and insecticide-treated bed nets (ITNs) were widely distributed in Southern Province, Zambia in 2007. Widespread indoor residual spraying has not been conducted in the study areas.

\section{Study design and methods}

Satellite images were used to construct a sampling frame for the random selection of households enrolled in prospective longitudinal and cross-sectional surveys [20]. The sampling frame for the random selection of households was constructed from a Quickbird ${ }^{\mathrm{TM}}$ satellite image obtained from DigitalGlobe Services, Inc. (Denver, Colorado). The image was imported into ArcGIS 9.2 (Redlands, CA) and locations of households were identified and enumerated manually. Structures of appropriate size and shape were identified as potential residences. Household locations of cases and controls were collected in the Universal Transverse Mercator, Zone 35 south coordinate system. Selected households were allocated to one of two study cohorts: longitudinal and crosssectional. Households in the longitudinal cohort were surveyed repeatedly approximately every other month and households in the cross-sectional cohort were surveyed once.

All individuals residing within a selected household were eligible to participate. After obtaining permission from the head of household and individual written informed consent, a questionnaire was administered to each participant older than 16 years of age residing within the household and to parents or guardians of those younger than 16 years of age. Data collected included demographic information, history of recent malaria and anti-malarial treatment, knowledge of malaria transmission and prevention, and the use of ITNs.

A blood sample was collected by finger prick for a rapid diagnostic test (RDT) for malaria and preparation of dried 


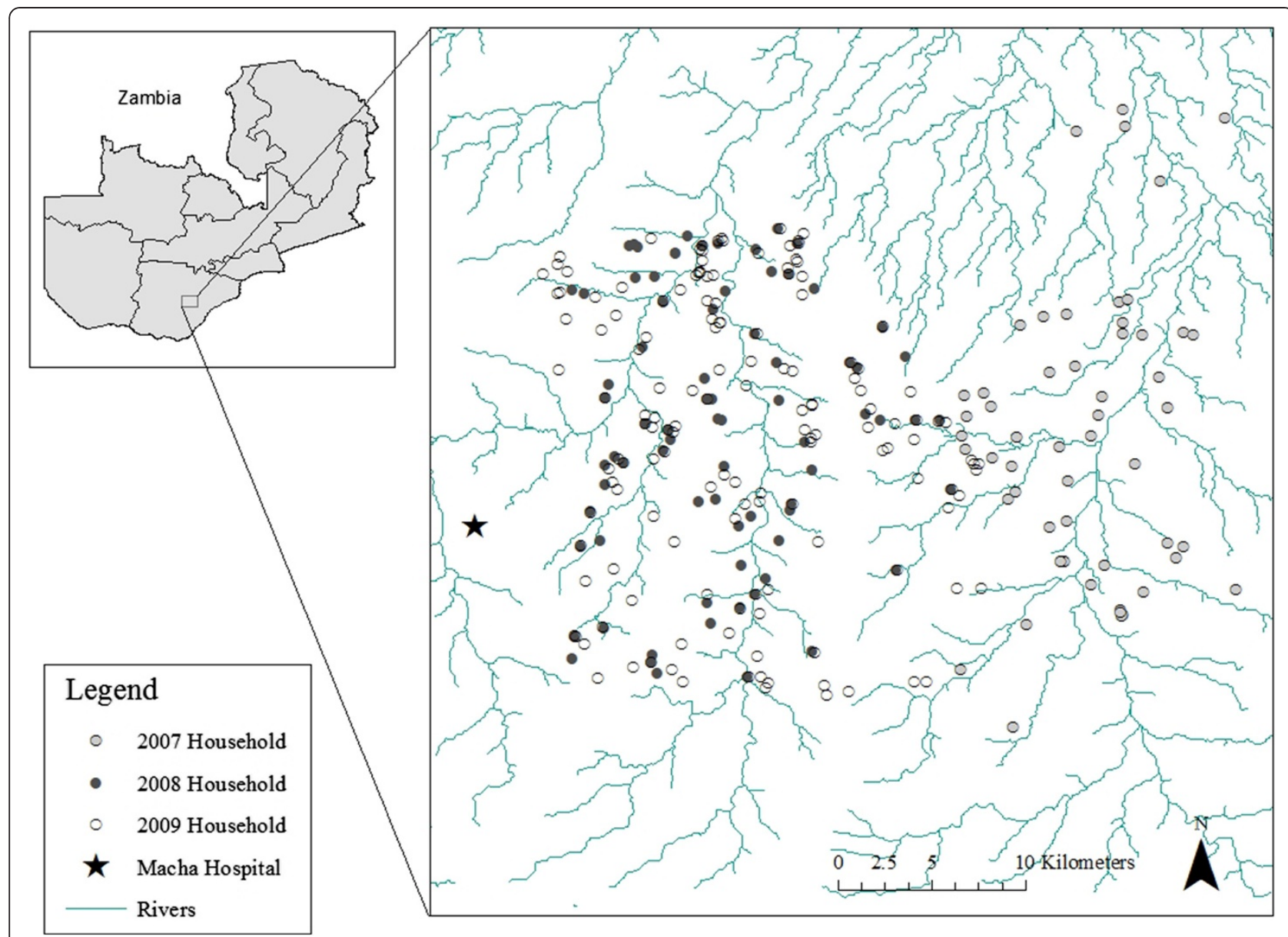

Figure 1 Location of study households in the 2007 and 2008-2009 study areas in Choma District, Southern Province, Zambia.

blood spots (DBS). The DBS were collected on filter paper (Whatman, Protein Saver card 903, Piscateway, New Jersey), dried overnight and stored individually with desiccant in a sealed plastic bag at $-20^{\circ} \mathrm{C}$. DBS collected in 2007 were stored with desiccant at room temperature and DBS collected from February to September in 2008 were initially stored at room temperature but subsequently stored at $-20^{\circ} \mathrm{C}$. All samples collected after September 2008 were stored at $-20^{\circ} \mathrm{C}$.

A RDT (ICT Diagnostics, Cape Town, South Africa) was used to detect P. falciparum histidine-rich protein 2 . This RDT was reported to detect $82 \%$ of test samples with $P$. falciparum at a concentration of 200 parasites $/ \mu \mathrm{L}$ and $98 \%$ of test samples with a concentration of 2000 parasites $/ \mu \mathrm{L}$, with false positives in $0.6 \%$ of negative samples [21]. Individuals who were RDT positive were offered treatment with artemether-lumefantrine $\left(\right.$ Coartem $\left.{ }^{\circledR}\right)$.

The study was approved by the University of Zambia Research Ethics Committee and the Institutional Review Board at the Johns Hopkins Bloomberg School of Public Health.

\section{Laboratory methods}

Antibodies to whole asexual parasite (NF54) lysate were measured by enzyme immunoassay using serum extracted from DBS. A circle of $7 \mathrm{~mm}$ (corresponding to approximately $10 \mu \mathrm{L}$ blood volume) in diameter was punched out of the DBS and half of the circle (approximately $5 \mu \mathrm{L}$ blood volume, therefore $2.5 \mu \mathrm{L}$ of serum) was used to extract serum. The half circle was soaked in $500 \mu \mathrm{L}(1: 200$ dilution of serum) 5\% skim milk in PBS containing $0.05 \%$ Tween 20 (PBST) for 1 hour at room temperature. Immulon 2HB flat-bottomed 96-well plates (Thermo, Rochester, New York) were coated with $1 \mu \mathrm{g} / \mathrm{mL}$ of whole $P$. falciparum asexual stage lysate diluted in PBS and incubated overnight at $4^{\circ} \mathrm{C}$. Plates were washed with PBST three times, blocked with 5\% skim milk in PBS for 1 hour at $37^{\circ}$ $\mathrm{C}$, and washed again with PBST four times. Eluted samples were plated in triplicate and incubated for 1 hour at $37^{\circ} \mathrm{C}$. Negative control serum (pooled serum from adults who had never been exposed to malaria) and positive control serum (serum from adults who resided in malaria endemic areas of Zimbabwe or Zambia) were diluted 1:200 
in 5\% skim milk PBST and included in triplicate on each plate. The plates were subsequently washed with PBST four times and incubated for one hour at $37^{\circ} \mathrm{C}$ with peroxidase-labeled goat anti-human IgG (KPL, Inc., Gaithersburg, Maryland). Following four washes, the plates were incubated for 15 minutes with $100 \mu \mathrm{L}$ of ABTS solution (KPL). Absorbance was measured at 405 $\mathrm{nm}$ and IgG levels were expressed as the optical density.

A threshold optical density (OD) value of 0.57 was established to distinguish seronegative from seropositive individuals based on the mean OD value plus three standard deviations from filter paper spotted with serum from ten individuals never exposed to malaria. Individuals with OD values above this threshold were defined as seropositive. Results were included only if the standard deviation of the triplicate values was within $20 \%$ of the mean OD value. The mean standard deviation for the triplicate values was 0.020 in 2008 and 0.026 in 2009 .

\section{Statistical methods for the longitudinal analysis}

Associations between RDT positivity and antibody levels to $P$. falciparum antigens, expressed as $\mathrm{OD}$ values and adjusted for age, were analyzed using data from participants enrolled in the longitudinal cohort. Malaria infection was defined in two ways: 1) a positive RDT result on the day of the study visit was defined as concurrent malaria; and 2) a positive RDT result at any previous study visit was defined as prior malaria. The change in antibody level over the one year study period was expressed as the mean difference in OD values between study visits. To account for within-subject correlation, a linear regression model was fitted adjusting for within subject correlation, with random intercept and robust standard errors.

\section{Statistical methods for the spatial analysis}

Using the coefficient for the change in the OD value in individuals with a prior documented history of recent malaria, participants enrolled in the longitudinal cohort in 2008 and 2009 were categorized as either a case or control. A case was defined as an individual whose change in OD value from the first to the last study visit at the end of the study year exceeded the coefficient from the linear model, and thus represented someone with a greater than expected increase in OD value and serological evidence of recent exposure to P. falciparum. A control was defined as an individual whose change in OD value from the first to the last study visit at the end of the study year did not exceed the coefficient from the linear model. Spatial clustering of cases was analyzed with SatScan ${ }^{\text {TM }}$ software (version 9.0) using the Kulldof spatial statistic [22] and a Bernoulli probability model. Coordinates were specified using a Cartesian coordinate system and the maximum spatial cluster size was set at $50 \%$ of the population at risk. To detect clusters, SatScan generated circular windows of different sizes. The circular window shape is the most commonly used shape $[23,24]$ and represents the most compact area. The number of cases in each window was compared to the expected number of cases based on the total population size and the total number of cases. The likelihood function was maximized over all scanned windows and the window with the maximum likelihood function was the cluster least likely to arise by chance. The p-value was obtained through Monte Carlo hypothesis testing with 999 simulations.

\section{Results}

\section{Baseline characteristics of participants}

Antibody levels to $P$. falciparum antigens were measured in 234 participants between April and July 2007 (crosssectional cohort $=90$, longitudinal cohort $=144), 435$ participants between February and December 2008 $($ cross-sectional cohort $=317$, longitudinal cohort $=118)$, and 855 participants between January and December 2009 $($ cross-sectional cohort $=675$, longitudinal cohort $=180)$. Most participants enrolled in the longitudinal cohort in 2008 continued to be followed in 2009, although three longitudinal households were replaced and six new households were added in 2009. The median age of the study participants was 12.2 years (interquartile range [IQR]: 5.08, 29.9) in 2007, 14.2 years (IQR: $6.34,34.2$ ) in 2008 , and 14.0 years (IQR: 6.34, 32.1) in 2009). Slightly less than half of the participants were male $(47 \%)$ (Table 1). The prevalence of RDT

Table 1 Characteristics of the study populations in 2007 and 2008-2009

\begin{tabular}{|c|c|c|c|c|c|c|}
\hline & \multicolumn{2}{|c|}{2007} & \multicolumn{2}{|c|}{2008} & \multicolumn{2}{|c|}{2009} \\
\hline & Cross-Sectional & $\begin{array}{l}\text { Longitudinal } \\
\left(1^{\text {st }} \text { visit }\right)\end{array}$ & Cross-Sectional & $\begin{array}{l}\text { Longitudinal } \\
\left.\text { (1 } 1^{\text {st }} \text { visit }\right)\end{array}$ & Cross- Sectional & $\begin{array}{l}\text { Longitudinal } \\
\left.\text { (1 } 1^{\text {st }} \text { visit }\right) \\
\end{array}$ \\
\hline Number of participants & 90 & 144 & 317 & 118 & 675 & 180 \\
\hline Median age in years (range) & $12.8(0.50-83)$ & $12.1(0.21-86)$ & $14.2(0.25-84)$ & $14.3(0.39-78)$ & $14.0(0.07-85)$ & $13.0(0.23-79)$ \\
\hline Male (\%) & $44(49)$ & $63(44)$ & $149(47)$ & $58(49)$ & $322(48)$ & $85(47)$ \\
\hline RDT positive (\%) & $29(32.2)$ & $37(25.7)$ & $31(9.78)$ & $4(3.39)$ & $10(1.48)$ & $2(1.11)$ \\
\hline
\end{tabular}


positivity differed markedly by study location and year: $24 \%$ of the baseline population (cross-sectional cohort plus the first visit of longitudinal cohort) was RDT positive in the eastern area in 2007, compared to $8.1 \%$ and $1.4 \%$ in the western area in 2008 and 2009, respectively.

\section{Seropositivity increased with age}

IgG antibody levels to $P$. falciparum were measured in the baseline study population consisting of participants in the cross-sectional cohort and the first visit of the longitudinal cohort. In each year, the proportion of seropositive individuals increased with age (Figure 2).
Consistent with the differences in parasite prevalence, the proportion of seropositive children younger than five years of age was lower in the 2008 and 2009 study areas than in the 2007 study area (34\% in 2007, $11 \%$ in 2008 and $12 \%$ in 2009).

\section{RDT positivity was positively correlated with higher serologic responses}

The effect of documented malaria during the study period on antibody levels to $P$. falciparum antigens was assessed among participants in the longitudinal cohort. In 2007, 114 individuals enrolled in the longitudinal cohort had at least two study visits. Of these, 47 had at
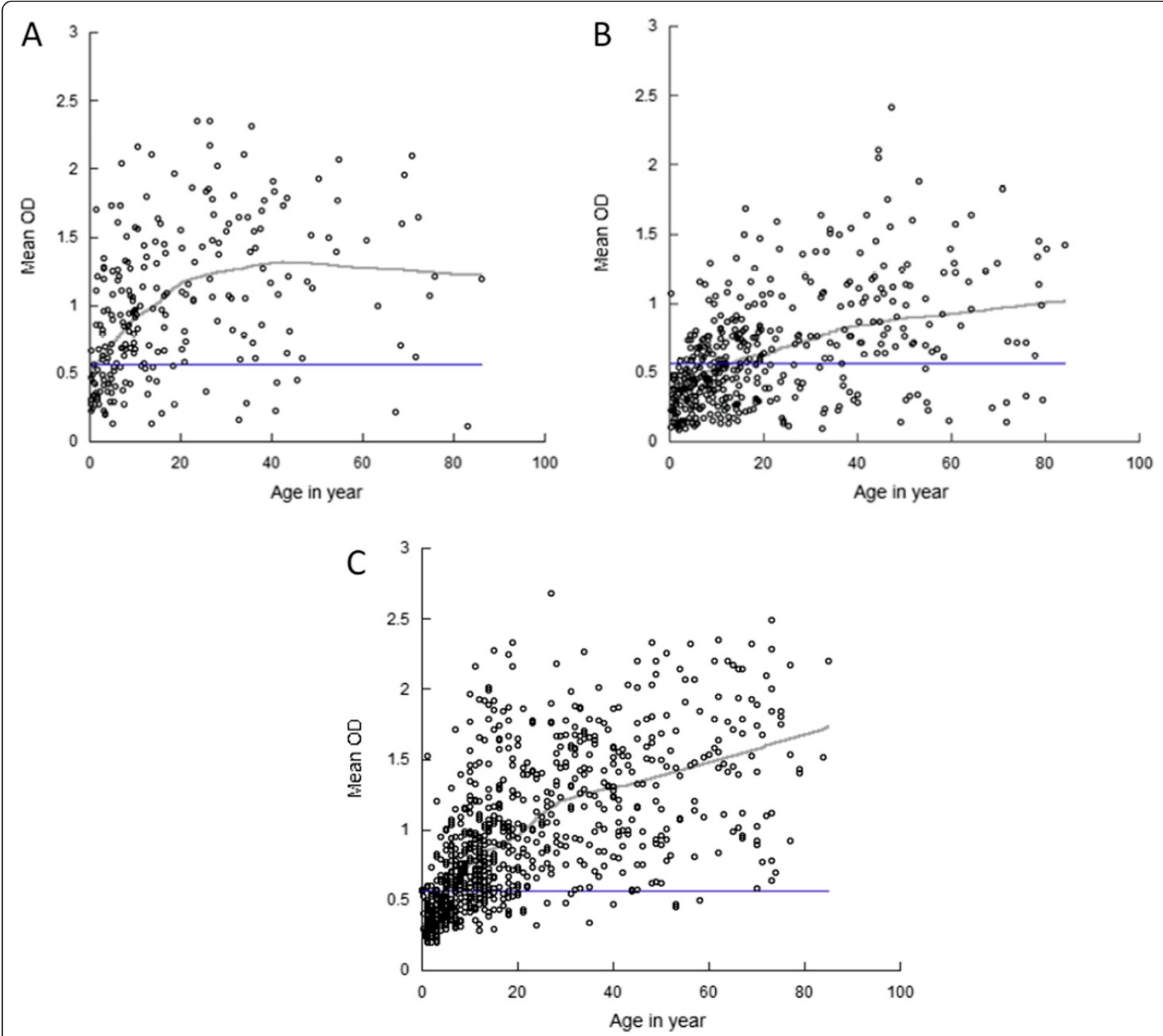

Figure 2 lgG antibody levels to P. falciparum whole antigen expressed as optical density values by age: A. 2007, B. 2008 and C. 2009. A Lowess curve was fit to the data. The straight line indicates the threshold OD value established using the mean OD value plus three standard deviations. 
least one RDT positive result, including 15 participants with two RDT positive results and two participants with three RDT positive results. To test the effect of having multiple RDT positive results during prior visits, the OD values at the end of 2007 study year among participants with a single positive RDT (mean $\mathrm{OD}=1.29$ ) was compared to participants with two or three RDT positive results (mean $\mathrm{OD}=1.09$; $p=0.66)$. As there was no statistically significant difference in mean EIA OD between individuals with a single or multiple RDT positive results, RDT results during any prior study visits were dichotomized as negative (no prior positive RDT result) or positive (at least one prior RDT positive result) for the analysis.

In 2008, 99 individuals enrolled in the longitudinal cohort had at least two study visits. Of these, nine participants had a single RDT positive result and one participant had two RDT positive results. In 2009, 168 individuals enrolled in the longitudinal cohort had at least two study visits. Only two were RDT positive and none had multiple RDT positive results. Due to the small number of RDT positive results in 2009, the association between RDT positivity and antibody levels to $P$. falciparum antigens was not examined.

The association between the antibody levels to $P$. falciparum antigens and a concurrent or prior documented episode of malaria was assessed, adjusting for age. In 2007, participants with a positive RDT result at the concurrent visit had a 0.177 higher OD value (95\% CI: $0.063,0.292, \mathrm{p}=0.002)$ than $\mathrm{RDT}$ negative participants, adjusting for age (Table 2). In 2008, participants with a positive RDT result at the concurrent visit had a statistically non-significant 0.063 lower OD value (95\% CI: $-0.245,0.119, \mathrm{p}=$ 0.51 ) than RDT negative participants after adjusting for age (Table 2). Participants with at least one positive RDT result at any prior visit during the study period had a 0.261 higher OD value (95\% CI: 0.090, $0.432, \mathrm{p}=0.003)$ in 2007 and a 0.116 higher OD value (95\% CI: $0.009,0.222, \mathrm{p}=0.03)$ in 2008 compared to RDT negative participants after adjusting for age (Table 2).
Spatial clustering of households comprised of individuals with serologic evidence of recent malaria

The coefficient for the OD value from the linear model for individuals having at least one positive RDT result at any prior visit during the study period in $2008(\beta=0.116)$, was used to assess whether serology could be used to identify spatial clusters of malaria transmission in the study area for 2008 and 2009. In 2008, 55 participants had samples collected at the beginning (February) and end (December) of the study period. The median age at the first study visit was 11 years (IQR: 6, 33) and the median interval between the first and the last study visit was 296 days (range: 284 to 305 days). Of these 55 participants, 43 (78\%) with a median age of 14 years (IQR: 7,36$)$ had OD values that increased more than 0.116 . In 2009, 74 individuals had samples collected at the beginning (January) and end (December) of the study period. The median age at the first study visit was 13 years (IQR: $5.6,35$ ) and the median interval between the first and last study visit was 305 days (range: 281 to 324 days). Of these 74 participants, $10(14 \%)$ with a median age of 21 years (IQR: 7.4, 30) had an OD values that exceeded 0.116.

Participants with OD values that exceeded 0.116 over the one year study period were classified as cases and clusters of households with cases were identified. The primary household clusters identified in 2008 (within a $9.2 \mathrm{~km}$ radius) were not statistically significant $(\mathrm{p}=0.15)$, but household clusters identified in 2009 (within a $5.4 \mathrm{~km}$ radius) achieved marginal statistical significance $(p=0.06)$ (Table 3$)$. Study households were overlaid on a malaria risk map generated from ecological characteristics of the terrain [20] to assess whether spatial clusters of recent malaria cases identified serologically were consistent with ecological predictors of malaria transmission in the study area (Figure 3). The cluster of households identified serologically in 2009 overlapped a region previously identified to be at risk for malaria transmission (Figure 3).

\section{Discussion}

Antibody levels to whole, asexual stage P. falciparum antigens measured by enzyme immunoassay increased with increasing age and were correlated with prior malaria infection as documented by RDT in this region

Table 2 Association between RDT positivity at the concurrent and prior visit and EIA OD values adjusting for age, 2007 and 2008

\begin{tabular}{lcccc}
\hline & \multicolumn{2}{c}{$\mathbf{2 0 0 7}$} & & $\mathbf{2 0 0 8}$ \\
\cline { 2 - 5 } & Coefficient & p-value & Coefficient & p-value \\
\hline Positive RDT result at the concurrent study visit & 0.177 & 0.002 & -0.063 & 0.50 \\
Positive RDT result at the prior study visit & 0.261 & 0.003 & 0.116 & 0.03 \\
\hline
\end{tabular}


Table 3 Spatial clusters of households with positive cases more than expected, 2008 and 2009

\begin{tabular}{lcccrr}
\hline Year & $\begin{array}{c}\text { Total population in } \\
\text { the cluster }\end{array}$ & $\begin{array}{c}\text { Observed number of } \\
\text { positive cases }\end{array}$ & $\begin{array}{c}\text { Expected number of } \\
\text { positive cases }\end{array}$ & Relative risk & p-value \\
\hline $\mathbf{2 0 0 8}$ & 27 & 25 & 21.11 & 1.44 & 0.15 \\
$\mathbf{2 0 0 9}$ & 34 & 9 & 4.59 & 10.59 & 0.06 \\
\hline
\end{tabular}

of declining malaria transmission in southern Zambia. In the year with the lowest parasite prevalence, spatial clustering of individuals with serologic evidence of recent infection overlapped with a region at risk for malaria transmission as previously determined by ecological factors [20]. These findings suggest that serology may be a useful tool for monitoring malaria transmission in regions of declining malaria burden with low levels of parasite prevalence.

Residents of malaria endemic areas acquire antibodies to $P$. falciparum and clinical immunity to malaria after repeated exposure, with earlier acquisition of seropositivity and clinical immunity in regions with higher transmission intensity [25]. Immunity to clinical malaria and seropositivity increase with increasing age due to cumulative exposure to malaria parasites; however, protective immunity may wane in the absence of repeated exposure to malaria parasites [26]. In southern Zambia, antibody levels demonstrated age-dependent increases consistent with other studies $[10,11]$. Although antibodies to whole parasite lysate were measured, prior reports demonstrated that antibody responses to whole schizont extract were comparable to antibody responses to single antigens such as AMA1 and MSP-1 19 [14].

The use of serology as a marker of malaria transmission intensity and recent exposure to P. falciparum was recently described [10]. A potential disadvantage of serology to track changes in malaria transmission is that antibody responses to $P$. falciparum may not be suitable to detect short term changes in population immunity in regions with rapid declines in malaria transmission. This study attempted to identify recent exposure to $P$. falciparum by measuring an increase in OD values above the expected value for having RDT positive result during one year study period. The findings suggest that recent exposure to $P$. falciparum can be detected through increases in OD values. Prior studies measured antibody responses to single parasite antigens, such as AMA1 or $\mathrm{MSP}_{19}$, and samples were collected in cross-sectional studies [9,11-13]. In contrast, we were able to measure serological responses in a longitudinal cohort and adjusted for within-individual heterogeneity.

Spatial clusters of individuals with serological evidence of recent infection was identified and these clusters were
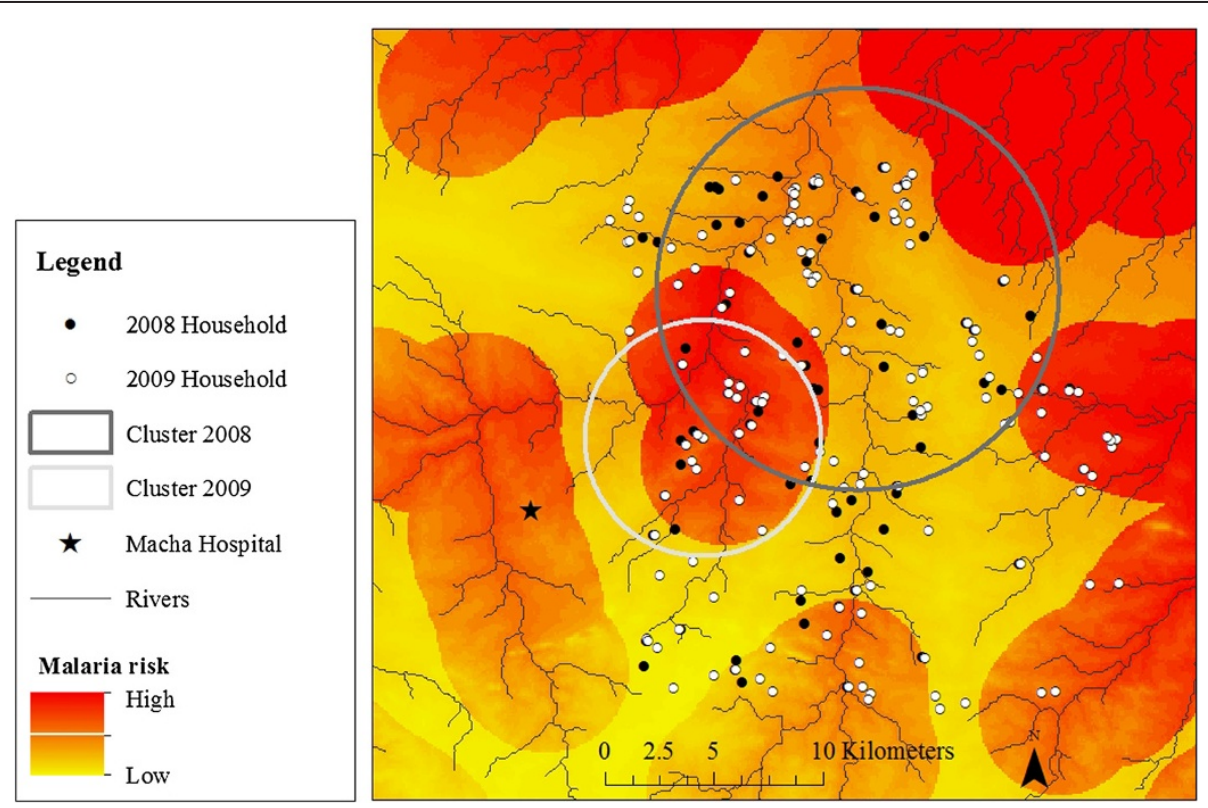

Figure 3 Spatial clusters of recent malaria cases identified serologically and ecological predictors of malaria transmission. Study households are shown in black (2008) and white (2009) dots. Spatial clusters of households comprising residents with serologic evidence of recent malaria infection are shown in the large circles ( $2008=$ grey; $2009=$ light grey). The background consists of a malaria risk map based on ecological features of the terrain [20], with the darker red indicating a higher risk of malaria. 
validated by comparing them to a previously reported malaria risk map of the study area [20]. Prior studies identified clusters of malaria transmission using serology but the reported clusters were smaller $(<1 \mathrm{~km})$ than the clusters identified in southern Zambia $(>5 \mathrm{~km})[27,28]$. The small sizes of the clusters were attributed to the estimated distance traversed by the mosquito vector [29]. In these studies, antibodies to specific antigens such as $\mathrm{MSP}_{19}$, AMA1 and MSP2 were measured; the broader antibody response to whole parasite lysate in our study may account for the larger cluster sizes.

\section{Conclusions}

Antibodies to whole, asexual stage $P$. falciparum antigens were positively correlated with prior RDT positivity. Serological surveys to whole asexual $P$. falciparum antigens using blood collected as dried blood spots can be used to detect recent malaria infection and identify focal areas of transmission, particularly in a region of declining malaria burden.

\section{Competing interests}

The authors declare that they have no competing interests.

\section{Authors' contributions}

TK participated in the coordination of the study, performed the data analysis and drafted the manuscript. SC performed the enzyme immunoassays and reviewed the manuscript. TS participated in the spatial analyses and reviewed the manuscript. $\mathrm{HH}$ coordinated the data and specimen collection and reviewed the manuscript. SM supervised the laboratory assays and reviewed the manuscript. PET supervised the field activities and reviewed the manuscript. GG participated in the spatial analyses and reviewed the manuscript. WJM conceived of the study, participated in its design and coordination, and participated in the preparation of the manuscript. All authors read and approved the final manuscript.

\section{Acknowledgements}

We thank the residents of Choma District who participated in the study, Dr. Nirbhay Kumar for provision of the samples for validation of the enzyme immunoassay, and Dr. Thomas Louis for statistical advice. This work was supported by the Johns Hopkins Malaria Research Institute and the Bloomberg Family Foundation.

\section{Author details}

${ }^{1}$ Department of Epidemiology, Johns Hopkins Bloomberg School of Public Health, Baltimore, MD, USA. ${ }^{2}$ Malaria Institute at Macha, Macha Research Trust, Choma, Zambia. ${ }^{3}$ Department of Molecular Microbiology and Immunology, Johns Hopkins Bloomberg School of Public Health, Baltimore, MD, USA.

Received: 2 October 2012 Accepted: 12 December 2012 Published: 31 December 2012

\section{References}

1. Barnes Kl, Chanda P, Ab Barnabas G: Impact of the large-scale deployment of artemether/lumefantrine on the malaria disease burden in Africa: case studies of South Africa, Zambia and Ethiopia. Malar J 2009, 8(Suppl 1):S8.

2. O'Meara WP, Mangeni JN, Steketee R, Greenwood B: Changes in the burden of malaria in sub-Saharan Africa. Lancet Infect Dis 2010, 10:545-555.

3. Chizema-Kawesha E, Miller JM, Steketee RW, Mukonka VM, Mukuka C, Mohamed AD, Miti SK, Campbell CC: Scaling up malaria control in Zambia: progress and impact 2005-2008. Am J Trop Med Hyg 2010, 83:480-488.
4. Government of the Republic of Zambia, Ministry of Health: Zambia national malaria indicator survey 2010. 2011. http://www.nmcc.org.zm/files/ FullReportZambiaMIS2010_001.pdf.

5. Steketee RW, Sipilanyambe N, Chimumbwa J, Banda JJ, Mohamed A, Miller J, Basu S, Miti SK, Campbell CC: National malaria control and scaling up for impact: the Zambia experience through 2006. Am J Trop Med Hyg 2008, 79:45-52.

6. World Health Organization: Malaria deaths decline by $66 \%$ in Zambia. Geneva: WHO; 2009.

7. World Health Organization: World malaria report 2010. Geneva: WHO; 2011.

8. Corran P, Coleman P, Riley E, Drakeley C: Serology: a robust indicator of malaria transmission intensity? Trends Parasitol 2007, 23:575-582.

9. Drakeley CJ, Corran PH, Coleman PG, Tongren JE, McDonald SL, Carneiro I, Malima R, Lusingu J, Manjurano A, Nkya WM, Lemnge MM, Cox J, Reyburn $H$, Riley EM: Estimating medium- and long-term trends in malaria transmission by using serological markers of malaria exposure. Proc Nat Acad Sci USA 2005, 102:5108-5113.

10. Drakeley C, Cook J: Potential contribution of sero-epidemiological analysis for monitoring malaria control and elimination: historical and current perspectives. Adv Parasitol 2009, 69:299-352.

11. Stewart L, Gosling R, Griffin J, Gesase S, Campo J, Hashim R, Masika P, Mosha J, Bousema T, Shekalaghe S, Cook J, Corran P, Ghani A, Riley EM, Drakeley C: Rapid assessment of malaria transmission using age-specific sero-conversion rates. PLOS One 2009, 4:e6083.

12. Cook J, Reid H, lavro J, Kuwahata M, Taleo G, Clements A, McCarthy J, Vallely A, Drakeley C: Using serological measures to monitor changes in malaria transmission in Vanuatu. Malar J 2010, 9:169.

13. Cook J, Kleinschmidt I, Schwabe C, Nseng G, Bousema T, Corran PH, Riley EM, Drakeley CJ: Serological markers suggest heterogeneity of effectiveness of malaria control interventions on Bioko Island, equatorial Guinea. PLoS One 2011, 6:e25137.

14. Akpogheneta OJ, Duah NO, Tetteh KK, Dunyo S, Lanar DE, Pinder M, Conway DJ: Duration of naturally acquired antibody responses to bloodstage Plasmodium falciparum is age dependent and antigen specific. Infect Immun 2008, 76:1748-1755.

15. Corran PH, Cook J, Lynch C, Leendertse H, Manjurano A, Griffin J, Cox J, Abeku T, Bousema T, Ghani AC, Drakeley C, Riley E: Dried blood spots as a source of anti-malarial antibodies for epidemiological studies. Malar J 2008, 7:195.

16. Estevez PT, Satoguina J, Nwakanma DC, West S, Conway DJ, Drakeley CJ: Human saliva as a source of anti-malarial antibodies to examine population exposure to Plasmodium falciparum. Malar J 2011, 10:104.

17. Chidi AP, Chishimba S, Kobayashi T, Hamapumbu H, Mharakurwa S, Thuma PE, Moss WJ: Validation of oral fluid samples to monitor serological changes to Plasmodium falciparum: an observational study in southern Zambia. Malar J 2011, 10:162.

18. Kent RJ, Thuma PE, Mharakurwa S, Norris DE: Seasonality, blood feeding behavior, and transmission of Plasmodium falciparum by Anopheles arabiensis after an extended drought in southern Zambia. Am J Trop Med Hyg 2007, 76:267-274.

19. Mharakurwa S, Thuma PE, Norris DE, Mulenga M, Chalwe V, Chipeta J, Munyati S, Mutambu S, Mason PR: Malaria epidemiology and control in Southern Africa. Acta Trop 2012, 121:202-206.

20. Moss WJ, Hamapumbu H, Kobayashi T, Shields T, Kamanga A, Clennon J, Mharakurwa S, Thuma PE, Glass G: Use of remote sensing to identify spatial risk factors for malaria in a region of declining transmission: a cross-sectional and longitudinal community survey. Malar J 2011, 10:163.

21. World Health Organization: Malaria rapid diagnostic test performance. Results of WHO product testing of malaria RDTs: round 1. 2008. http://www.who.int/ tdr/publications/tdr-research-publications/rdt3_summary.pdf.

22. Kulldorff $M$, Nagarwalla N: Spatial disease clusters: detection and inference. Stat Med 1995, 14:799-810.

23. Haque U, Hashizume M, Sunahara T, Hossain S, Ahmed SM, Haque R, Yamamoto T, Glass GE: Progress and challenges to control malaria in a remote area of Chittagong hill tracts, Bangladesh. Malar J 2010, 9:156.

24. Loha E, Lunde TM, Lindtjorn B: Effect of bednets and indoor residual spraying on spatio-temporal clustering of malaria in a village in South ethiopia: a longitudinal study. PLoS One 2012, 7:e47354.

25. Doolan DL, Dobano C, Baird JK: Acquired immunity to malaria. Clin Microbiol Rev 2009, 22:13-36. Table of Contents. 
26. Ghani AC, Sutherland CJ, Riley EM, Drakeley CJ, Griffin JT, Gosling RD, Filipe JA: Loss of population levels of immunity to malaria as a result of exposure-reducing interventions: consequences for interpretation of disease trends. PLoS One 2009, 4:e4383.

27. Bousema T, Drakeley C, Gesase S, Hashim R, Magesa S, Mosha F, Otieno S, Carneiro I, Cox J, Msuya E, Kleinschmidt I, Maxwell C, Greenwood B, Riley E, Sauerwein R, Chandramohan D, Gosling R: Identification of hot spots of malaria transmission for targeted malaria control. J Infect Dis 2010, 201:1764-1774.

28. Bejon P, Williams TN, Liljander A, Noor AM, Wambua J, Ogada E, Olotu A, Osier FH, Hay SI, Farnert A, Marsh K: Stable and unstable malaria hotspots in longitudinal cohort studies in Kenya. PLoS Med 2010, 7:e1000304.

29. Bousema T, Griffin JT, Sauerwein RW, Smith DL, Churcher TS, Takken W, Ghani A, Drakeley C, Gosling R: Hitting hotspots: spatial targeting of malaria for control and elimination. PLoS Med 2012, 9:e1001165.

doi:10.1186/1475-2875-11-438

Cite this article as: Kobayashi et al:: Temporal and spatial patterns of serologic responses to Plasmodium falciparum antigens in a region of declining malaria transmission in southern Zambia. Malaria Journal 2012 11:438.

\section{Submit your next manuscript to BioMed Central and take full advantage of:}

- Convenient online submission

- Thorough peer review

- No space constraints or color figure charges

- Immediate publication on acceptance

- Inclusion in PubMed, CAS, Scopus and Google Scholar

- Research which is freely available for redistribution 